\title{
ENHANCED MULTIQUERY SYSTEM USING KNN FOR CONTENT BASED IMAGE RETRIEVAL
}

\author{
Meenu (1), Sonika Jindal (2) \\ (1) Research Scholar, Department of Computer Science Engineering, SBSSTC, Ferozepur \\ kalrameenu1991@gmail.com \\ (2) Assistant Professor, Department of Computer Science Engineering, SBSSTC, Ferozepur \\ sonikamanoj@gmail.com
}

\begin{abstract}
Content Based Image Retrieval (CBIR) techniques are becoming an essential requirement in the multimedia sys tems with the widespread use of internet, declining cost of storage devices and the exponential growth of un-annotated digital image information available in recent years. Therefore multi query systems have been used rather than a single queryin order to bridge the semantic gaps and in order to understand user's requirements. Mo reover, query replacement algorithm has been used in the previous works in which user provides multiple images to the query image set referred as representative images. Feature vectors are extracted for each image in the representative image set and every image in the database. The centroid, Crep of the representative images is obtained by computing the mean of their feature vectors. Then every image in the representative image set is replaced with the same candidate image in the dataset one by one and new centroids are calculated for every replacement. The distance between each of the centroids resulting from the replacement and the representative image centroid Crep is calculated using Euclidean distance. The cumulative sum of these distances determines the similarity of the candidate image with the representative image set and is used for ranking the images. The smaller the distance, the similar will be the image with the representative image set. But it has some research gaps like it takes a lot of time to extract feature of each and every image from the database and compare our image with the database images and complexity as well as costincreases. So in our proposed work, the KNN algorithm is applied for classification of images in the database image set using the query images and the candidate images are reduced to images returned after classification mechanism which leads to decrease the execution time and reduce the number of iterations. Hence due to hybrid model of multi query and KNN, the effectiven ess of image retrieval in CBIR system increases. The language used in this work is C /C++ with Open CV libraries and IDE is Visual studio 2015. The experimental results show that our method is more effective to improve the performance of the retrieval of images.
\end{abstract}

\section{Keywords}

Content based image retrieval (CBIR), KNN, Multiquery, image database

\section{INTRODUCTION}

Content-Based Image Retrieval (CBIR) systems are search engines for image databases, which index images according to their content. A typical task solved by CBIR systems is that a user submits a query image or series of images and the system is required to retrieve images from the database as similar as possible. Another task is a support for browsing through large image databases, where the images are supposed to be grouped or organized in accordance with similar properties. Although the image retrieval has been an active research area for many years this difficult problem is still far from being solved. There are two main reasons, the first is so called semantic gap, which is the difference between information that can be extracted from the visual data and the interpretation that the same data have for a user in a given situation. The other reason is called sensory gap, which is the difference between a real object and its computational representation derived from sens ors, which measurements are significantly influenced by the acquisition conditions.. The feature vectors of images in the database form a feature database. The retrieval process is initiated when a user queries the system using an example image or sketch of the object. The query image is converted into the internal representation of feature vector using the same feature extraction routine that was used for building the feature database. The similarity measure is employed to calculate the distance between the feature vectors of query image and those of the target images in the feature database. Finally, the retrieval is performed using an indexing scheme which facilitates the efficient searching of the image database. Recently, user's relevance feedback is also incorporated to further improve the retrieval process in order to produce perceptually and semantically more meaningful retrieval results.

\section{RELATED WORK}

Sawas A. Chatzichristofis et al. (2008) deals with a new low level feature that is extracted from the images and can be used for indexing and retrieval. This feature is called "Color and Edge Directivity Descriptor" and incorporates color and texture in formation in a histogram. CEDD size is limited to 54 bytes per image, rendering this descriptor suitable for use in large image databases.

Khadidja et al. (2013) focused on CBIR and basic concepts pertaining to it, as well as Relevance Feedback and its various mechanisms. An important contribution in this work is a comparative analys is of CBIR systems using reference feedback: major models and approaches are discussed in detail from early heuristic methods to recently optimal learning algorithms, with more emphasize on their advantages and weaknesses.

Bhavneet Kaur et al. (2014) used the OPEN CV platform since it provides a C interface to implement various image processing algorithms. The work merges the feature extraction technique with this most suitable platform avail able for image algorithms. They have also computed the performance of the technique used in terms of various parameters like execution time, rotation, detect ability, accuracy, etc. 
Komal Juneja et al. (2015) presented a survey on low-level feature description techniques for Content Based Image Retrieval is presented with its various applications. In the modern era, with the explosive growth of image databases, huge amount of image and video archive led to rise of a new research and development of efficient method to searching, locating and retrieving of image. For this purpose, an efficient tool for searching, locating and retrieval of image is requi red.

Ghanshyam Raghuwanshi et al. (2015) proposes a novel technique for texture image retrieval based on tetrolet transforms. Tetrolets provide fine texture information due to its different way of analysis. Tetrominoes are applied at each decomposition level of an image and best combination of tetrominoes is selected, which better shows the geometry of an image at each level. All three high pass components of the decomposed image at each level are used as input values for feature extraction.

Jayant Mankar et al. (2016) states every day an enormous amount of data is retrieved and transmitted on the Internet. Internet gives rise to have the relevant information more quickly. Most of the users or researchers required the image data from the available image database. For the retrieval of concern image data from the huge database is tedious task in terms of the storage and retrieval time.

Garima Gupta et al. (2016) implemented a novel method for CBIR using Hough Transform, DCD and DWT feature with Support vector machine (SVM) as a classifier. In the process of feature extraction, firstly extract texture feature using discrete wavelet transform (DWT), extract color feature using dominant color descriptor (DCD) on RGB and HSV color space for improving computation and efficiency and for line detection use Hough Transform of images.

Lakhdar et al. (2016) presents an efficient region based image retrieval method, which uses multi-features color, texture and edge descriptors. In contrast to recent image retrieval methods, which use discrete wavelet transform (DWT), they proposed using shape adaptive discrete wavelet transform (SA-DWT).

Abbas $\mathrm{H}$ et al. (2016) studied the influence on performance of reducing the colors number contained in images. Accomplishing this task poses an extra overhead on the system, which requires more computation time, but, on the other hand, can accelerate the comparison process.

Aman Saini et al. (2016) evaluates the performance of CBIR system with parameters precision, recall, NMRR and retrieval time by using feature extraction techniques based upon colour - histogram, texture - GLCM and region -boundary descriptors of an image. NMRR and retrieval time of different query images have been evaluated.

Snehal Yadav et al. (2016) states that the community networking sites like Facebook as well as Flicker allow users to upload the images. E-com merce web sites like flipkart and amazon etc. also provide number of products related images to user. Images which are present in social networking websites are accompanied by various tags, comments, annotations and other related information to form an image-rich information networks.

Suchita Barkund et al. (2016) discusses that face identification introduces a challenging problem in the various domain areas like image analys is and computer vision. The number of the images available on internet among them most of the images is human facial images. Sometimes images not properly tagged with right names. So, Search-Based Face Annotation (SBFA) framework uses Content-Based Image Retrieval (CBIR) technique in mining web facial images for annotation.

Jagbir Singh Gill et al. (2016) explains the basis of presenting this research work is the retrieval of images based on the color and text components pres ent in the query image. The proposed work presents matching assessment of trademark or logo images using color features based over the index of Hue, Saturation and intensity. Overall, the work experimentally compared the XYZ and HSV algorithm on the measurement of Euclidean distance for Content Based Image retrieval.

\section{MOTIVATION OF THE WORK}

In the last two decades, CBIR systems have been improved a lot. However, there still remain some problems which have not been answered satisfactorily. First and foremost, problem is of semantic gap, which exist between low level feature representation of images and the actual visual perception of the image. Researchers all over the globe are working in the direction of narrowing down this semantic gap. Semantic gap is a big problem which can be seen as a collection of many small problems. The semantic gap is the lack of coincidence between the in formation that one can extract from the visual data and the interpretation that the same data has for a user in a given situation. Negative images which user don't want in the output as a result. In another word, images with high low-level feature similarities maystill be different in terms of user perception. So, similarity by low-level features, not always mean semantic similarity of these images. As number of images present in the database may be in large quantity, it takes a lot of time to feature each and every image from the database and compare our image with the database images. Every image has to be compared with images present in the database, so it increases the complexity of the system. If there are more number of comparisons, more hardware is required and thereby increasing the cost. In this work, we have identified such problems and tried to provide an effective solution to these problems.

\section{OBJECTIVES}

This paper is devoted to improving existing techniques involved in feature extraction, similarity matching and reducing the overall computation time of image retrieval system while increasing the accuracy. The main contributions of the thesis are listed below:

- Design and development of a multistage model for image retrieval to improve the retrie val accuracy by filtering down irrelevant images at each stage. 
- The accuracy of region based image retrieval system is improved by introducing novel region

- To reduce the number of iterations by using the image classification mechanism

- To reduce the processing time of the algorithm, thereby improving the overall efficiency of the system.

- To implement the proposed algorithm in OpenCV environment and evaluate the performance with the existing algorithm

\section{KNN}

An instance based learning method called the K-Nearest Neighbor or K-NN algorithm has been used in manyapplications in areas such as data mining, statistical pattern recognition, image processing. Successful applications include recognition of handwriting, satellite image and EKG pattern. In data mining, we often need to compare samples to see how similar they are to each other. For samples whose features have continuous values, it is customary to consider samples to be similar to each other if the distances between them are small. Other than the most popular choice of Euclidean distance, there are of course many other ways to define distance. The k-means clustering algorithm attempts to split a given anonymous data set (a set containing no information as to class identity) into a fixed number (k) of clusters.

Initially k number of so called centroids are chosen. A centroid is a data point (imaginary or real) at the center of a cluster. In Praat each centroid is an existing data point in the given input data set, picked at random, such that all centroids are unique (that is, for all centroids ci and $\mathrm{cj}, \mathrm{ci} \neq \mathrm{cj}$ ). These centroids are used to train a KNN classifier. The resulting cla ssifier is used to classify (using $k=1$ ) the data and thereby produce an initial randomized set of clusters. Each centroid is thereafter set to the arithmetic mean of the cluster it defines. The process of classification and centroid adjustment is repeated until the values of the centroids stabilize. The final centroids will be used to produce the final classification/clustering of the input data, effectively turning the set of initially anonymous data points into a set of data points, each with a class identity.

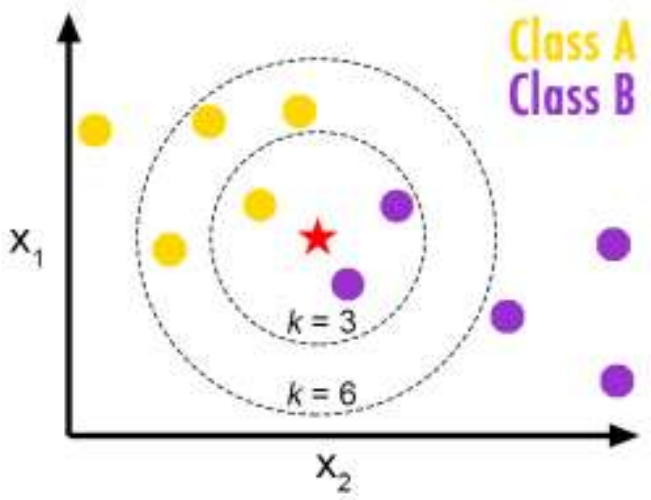

Figure 1. KNN Classification

The training examples are vectors in a multidimensional feature space, each with a class lab el. The training phase of the algorithm consists only of storing the feature vectors and class labels of the training samples.

In the classification phase, $\mathrm{k}$ is a user-defined constant, and an unlabeled vector (a query or test point) is classified by assigning the label which is most frequent among the k training samples nearest to that query point.

\section{IMPROVED MULTI QUERY CBIR USING QUERY REPLACEMENT}

A multi query system using query replacement algorithm was used in the previous work which utilizes the statistical features of a query image set to determine the similarity of the candidate images in the database for ranking and retrieval. Using this method with smaller number of query images, high retrieval precision rate is obtained but the major drawback is high computation cost during the run time. In this work a novel query replacement algorithm using KNN is proposed for boosting the efficiency of a multi query CBIR system. First of all, the database images are fetched and are classified using $\mathrm{KNN}$ algorithm based on the query images. The database is reduced to the classified images. The algorithm is based on the principle that if an element in set $\mathrm{X}$ is to be replaced with an element in set $\mathrm{Y}$, it will cause minimum information change if the replaced element has high similarity with the element being replaced.

- User provides $\mathrm{R}$ images to the query image set referred as representative images and $\mathrm{D}$ images in the database images referred as candidate images.

- Feature vectors are extracted for each image in the representative image set and every image in the database.

- $\quad$ The KNN algorithm is applied for classification of images in the database image using the query images.

- The candidate images are reduced to images returned after classification mechanism.

- The centroid, Crep of the representative images is obtained by computing the mean of their feature vectors. 
- Then every image in the representative image set is replaced with the same candidate image in the dataset one by one and new centroids are calculated for every replacement.

\section{PROPOSED ALGORITHM}

Input:

1. The $\mathrm{R}$ representative images with their feature vectors;

$$
X=\{x 1, x 2, \ldots, x R\} .
$$

2. $M$ candidate images in the database with their feature vectors;

$$
\mathrm{Y}=\{\mathrm{y} 1, \mathrm{y} 2, \ldots . \mathrm{yM}\} \text {. }
$$

Output:

Distances D1...M; the cumulative distance between Crep and the R Ci's.

Begin

Find the centroid, Crep, of the representative images

Apply KNN on candidate images ( $\mathrm{Y}$ ).

Remove the unclassified images from the candidate set

For $\mathrm{j}=1$ to $\mathrm{M}$

$\mathrm{Dj}=0$;

For $\mathrm{i}=1$ to $\mathrm{R}$

Replace $x i$ in $X$ with $y j$ in $Y$ so that $X^{\prime}=\{y j, x 2, \ldots, x R\}$.

Find centroid Ci' of the new $X$ '.

di = Euclidean distance (Crep, $\left.\mathrm{Ci}^{\prime}\right)$.

$\mathrm{Dj}=\sum \mathrm{di}$

End

End

End

\section{EXPERIMENTAL RESULTS}

Multiple number of experiments have been conducted on different categories of images. The different categories like animals, mountains, flowers, cars etc. have been used for testing and analysis. A retrieved image is considered to be correct if and only if it is in the same category as the query. For each query, a preselected number of images are retrieved which are illustrated and listed in the ascending order of the distance between the query and the retrieved images. The experiments are carried out in a personal computer with Intel Core 2 Duo processor with 2GB RAM. The program is developed using OpenCV libraries and Visual Studio IDE.

Each category image from the database was used in turn as a query with the scope set as $10,20,40,60,80,100$ and 200. After performing all the retrievals, the results are evaluated. Number of iterations, processing time, precision and recall are evaluated for the existing work and proposed work. Results from using the multi query replacement algorithm without KNN and with KNN are represented by using bar charts and tables. For the particular Query Image A, B, C, D, the cumulative sum of all the distances is computed. The system retrieved images according to the user's preference or the query images provided by the user. This attribute is useful to find particular kinds of images, for which no exact query can be found. Searching can be performed on an image that are somewhat similar. Figure 5.2 shows the matching of the database image with the query image. 


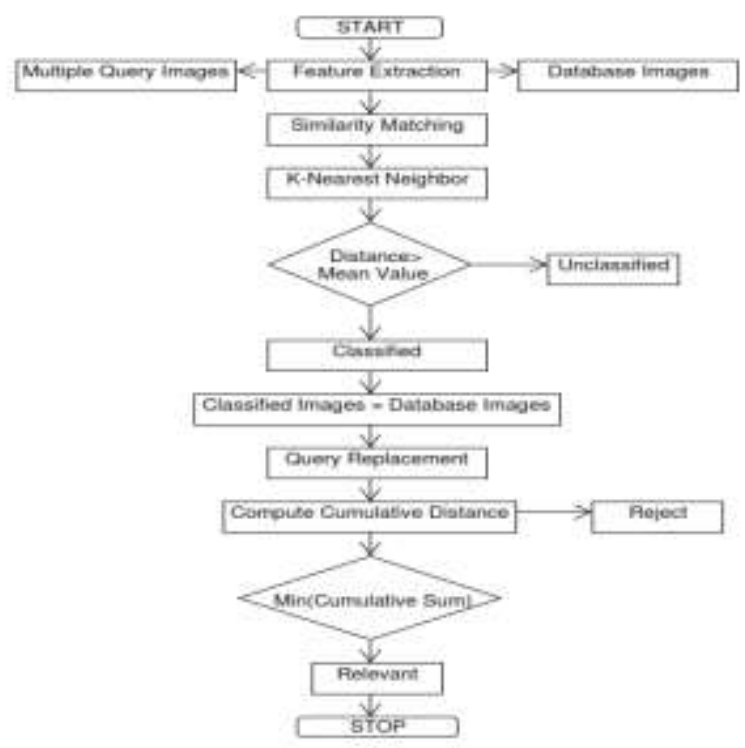

Figure 2. How Chart of Proposed Work

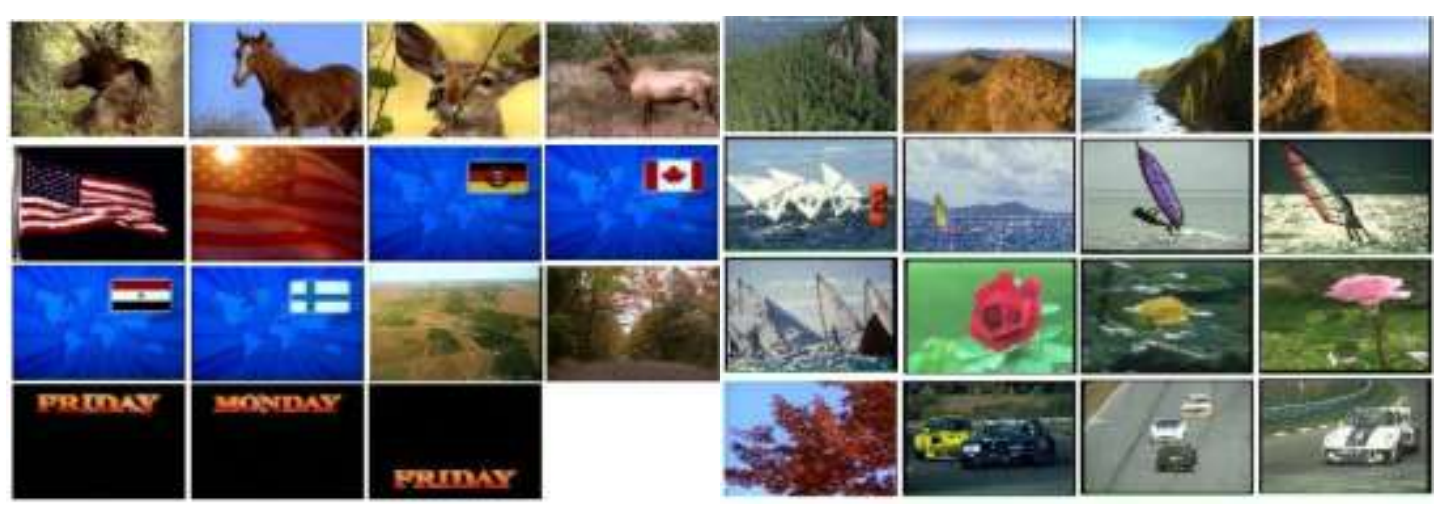

Figure 3. Different Categories of Images
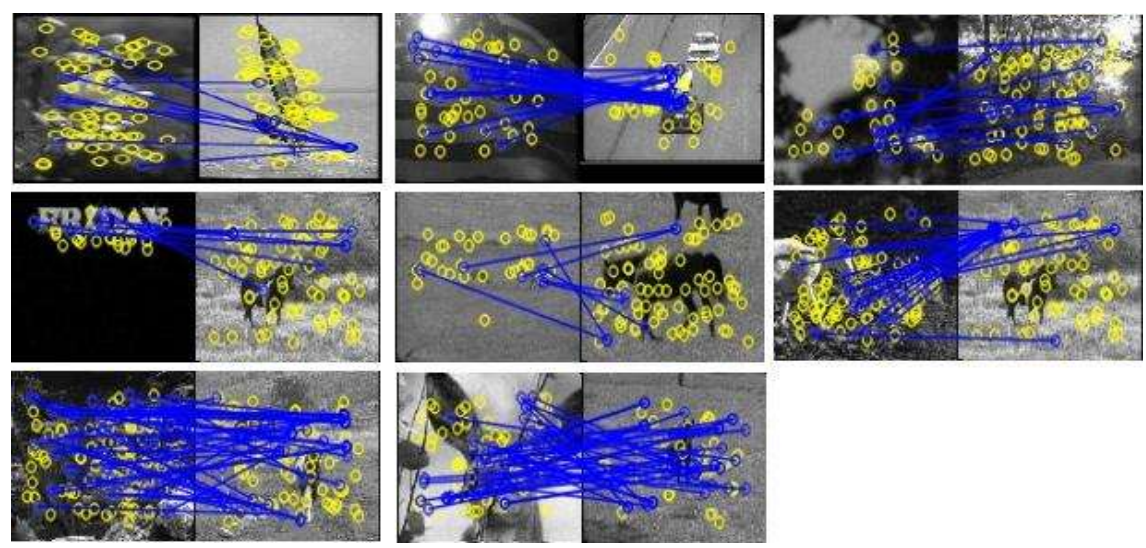

Figure 4. Similarity Matching of Query Image with Database Images

\section{PERFORMANCE EVALUATION}

The performance of a retrieval system is evaluated based on several criteria. Some of the commonly used performance measures are average precision, average recall, average retrieval rate. All these parameters are computed using precision and recall values computed for each query image. The precision of the retrieval is defined as the fraction of the retrieved images that are indeed relevant for the query:

$$
\text { Precision }=\frac{\text { No of relevant images retrieved }}{\text { Total no of images from the database }}
$$

The recall is the fraction of relevant images that is returned by the query: 


$$
\text { Recall }=\frac{\text { No of relevant images retrieved }}{\text { Total no of relevant images in the database }}
$$

A good retrieval system should have high values for precision and recall. Different set of images are taken in each experiment and the number of multiple queryimages are also different for some of the experiments. The best cumulative sum is carried out using the multi query replacement algorithm. KNN algorithm can be used for classification. Before replacing the query image with all the database images one by one, we should classify the database images into 2 groups: relevant and irrelevant. KNN uses the query images for classifying the database images. More relevant images are grouped into one container and the remaining are grouped into another container. As shown in the table 2 , out of 38 total images in the first experiment, 20 images are relevant and 18 are irrelevant. Now the database is reduced to 20 from 38. Only 20 images will be further used for multi query replacement algorithm which will further decreases the number of iterations and the processing time.

Table 1. KNN Classification in proposed work

\begin{tabular}{|c|c|c|c|c|c|c|c|}
\hline S.No. & $\begin{array}{c}\text { Total } \\
\text { Images }\end{array}$ & $\begin{array}{l}\text { Query } \\
\text { Images }\end{array}$ & $\begin{array}{c}\text { KNN } \\
\text { Classified }\end{array}$ & Unclassified & $\begin{array}{c}\text { Positive } \\
\text { In Final } \\
\text { Result }\end{array}$ & $\begin{array}{l}\text { Negative In } \\
\text { Final Result }\end{array}$ & $\begin{array}{c}\text { Total } \\
\text { Positive } \\
\text { Images In } \\
\text { Dataset }\end{array}$ \\
\hline 1 & 38 & 4 & 20 & 18 & 18 & 2 & 28 \\
\hline 2 & 22 & 3 & 10 & 12 & 6 & 4 & 16 \\
\hline 3 & 18 & 3 & 7 & 11 & 7 & 0 & 15 \\
\hline 4 & 27 & 3 & 12 & 15 & 12 & 0 & 24 \\
\hline 5 & 33 & 3 & 16 & 17 & 11 & 5 & 22 \\
\hline 6 & 44 & 2 & 23 & 21 & 23 & 0 & 44 \\
\hline 7 & 20 & 3 & 11 & 9 & 11 & 0 & 15 \\
\hline 8 & 15 & 2 & 9 & 6 & 6 & 3 & 12 \\
\hline
\end{tabular}

In the table 2, we have calculated the precision and recall for the same set of images that are used in table 1 . The precision is ranging from 0.67 to 1.0 which is a sign of improvement over the existing work. The number of iterations have been reduced substantially. The overall execution time is computed in milliseconds and has been reduced in comparison with existing work.

Table 2. Precision and Recall of Proposed Work

\begin{tabular}{|c|c|c|c|c|c|}
\hline S.No. & Precision & Recall & $\begin{array}{c}\text { Execution } \\
\text { Time }\end{array}$ & $\begin{array}{c}\text { Total } \\
\text { Iterations }\end{array}$ & $\begin{array}{c}\text { Best } \\
\text { Cumulative } \\
\text { Distance }\end{array}$ \\
\hline 1 & 0.90 & 0.64 & 11334 & 80 & 396 \\
\hline 2 & 0.60 & 0.38 & 3905 & 30 & 408 \\
\hline 3 & 1.00 & 0.47 & 3053 & 21 & 384 \\
\hline 4 & 1.00 & 0.50 & 5236 & 36 & 399 \\
\hline 5 & 0.69 & 0.50 & 5541 & 48 & 309 \\
\hline 6 & 1.00 & 0.52 & 4928 & 46 & 176 \\
\hline 7 & 1.00 & 0.73 & 3139 & 33 & 222 \\
\hline 8 & 0.67 & 0.50 & 2130 & 18 & 226 \\
\hline
\end{tabular}

Figure 5. illustrates the bar chart comparison for the existing work and the proposed work. After applying the KNN classification mechanism the number of iterations has been reduced in all the experiments. The database contains the multiple set of images that are relevant and irrelevant to us. Only the relevant images should be used for multi query replacement algorithm. 


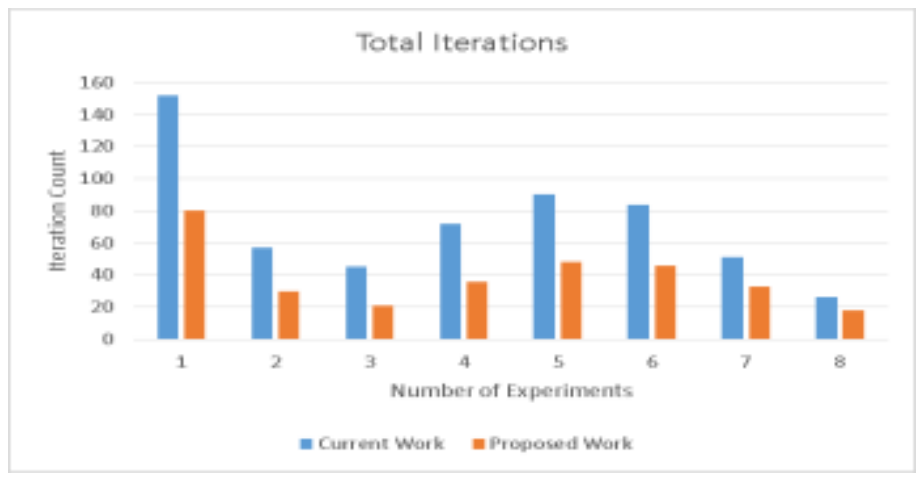

Figure 5. Iterations Bar Graph

Figure 6. shows the significance improvement in the execution time for the proposed work. The execution time has been reduced because we have reduced the database images after applying the KNN classification mechanism. There is an improvement of approximately $30 \%$ in overall execution time.

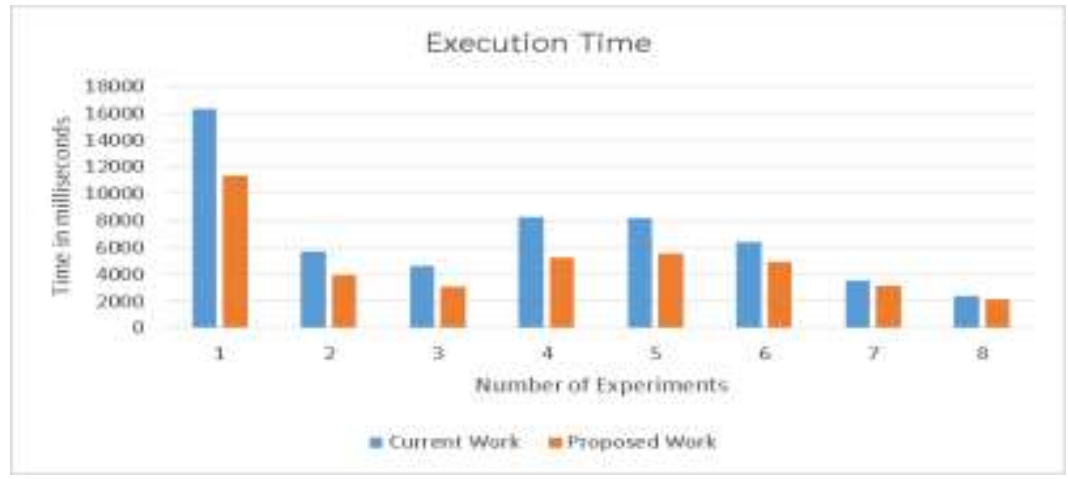

Figure 6. Execution Time bar Graph

Figure 7. illustrates the improvement in precision when KNN classification is applied on multi query replacement mechanism. After applying the classification using KNN, the precision of the proposed work has been increased and thereby increasing the overall efficiency of the system. A good CBIR system should have higher value of precision.

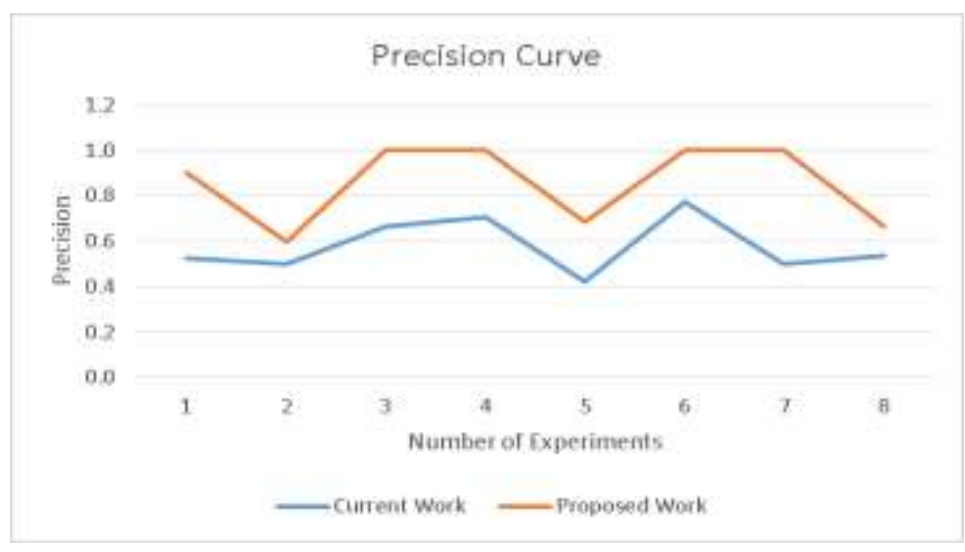

Figure 7. Precision comparison of Current and Proposed Work

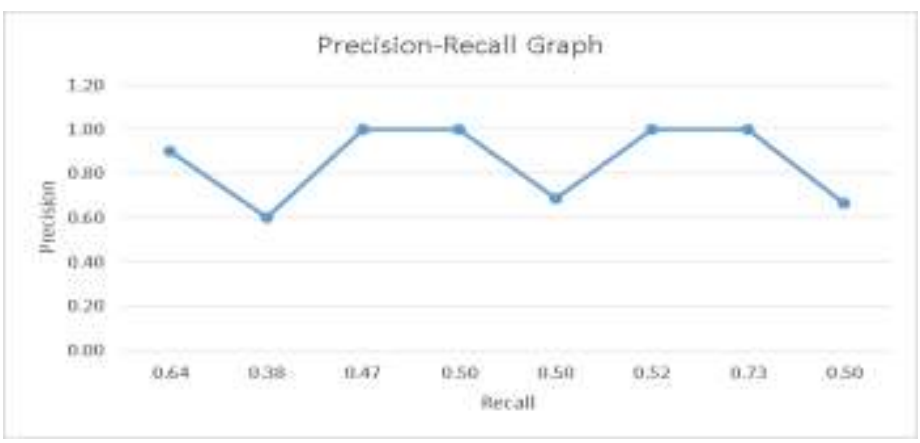

Figure 8. Precision-Recall Graph of proposed work 
In the figure 8, we have illustrated the precision-recall graph of the proposed work. For a given experiment, as the size increases, both the Recall and precision increased. This is expected as the number of positive and negative images can only remain constant or increase. The trade-off between the two values is governed by the size used.

\section{CONCLUSION}

We have reviewed the main components of a content based image retrieval system by applying KNN with multiple query replacement mechanism, including image feature representation, indexing, query processing, and query-image matching and user's interaction, while highlighting the current state of the art and the key -challenges. It has been acknowledged that it remains much room for potential improvement in the development of content based image retrieval system due to semantic gap between image similarity outcome and user's perception. Contributions of soft-computing approaches and natural language processing methods are especially required to narrow this gap.

For our investigation, it was necess ary to survey performance evaluation techniques. It was noted that those sample size factors are often omitted in CBIR performance meas ures. Their proven impact em phasizes the need to illus trate them for a proper representation of a system. This can be overcome by more in-depth analysis of results beyond the standard measures used as well as normalization procedures with respect to scope and number of irrelevant semantic classes. In the future scope, we can extend the multi query replacement mechanism with another available classifier and find out the differences between the present work.

\section{REFERENCES}

[1] J. Mankar, A. Sahu, K. Harode, M. Pawase, N. Titarmare, R. Karadbhajne and S. Katti, "CBIR Based on Color and Texture Features using DCT and DWT," International Journal of Engineering and Advanced Research Technology (IJEART) , pp. 60-62, 2016.

[2] G. Gupta and Manish Dixit, "CBIR on Biometric Application using Hough Transform with DCD ,DWT Features and SVM Classification," International Journal of Engineering and Innovative Technology (IJEIT), pp. 46 -50, 2016.

[3] R. Jain, S. Kumar Sinha and M. Kumar, " A New Image Retrieval System Based on CBIR," International Journal of Emerging Technology and Advanced Engineering , pp. 101-107, 2015.

[4] T. M. Rao , S. Setty and Y. Srinivas , "An Efficient System for Medical Image Retrieval using Generalized Gamma Distribution," I.J. Image, Graphics and Signal Processing, pp. 52-28, 2015.

[5] L. BELHALLOUCHE, K. BELLOULATA and K. KPALMA, "A New Approach to Region Based Image Retrieval using Shape Adaptive Discrete Wavelet Transform," I.J. Image, Graphics and Signal Processing,, pp. 1-14, 2016.

[6] Abbas H. Hassin Alasadi and Saba Abdual Wahid, "Effect of Reducing Colors Number on the Performance of CBIR System," I.J. Image, Graphics and Signal Processing,, pp. 10-16, 2016.

[7] K. Seetharaman and R. Shekhar, "COLOR IMAGE RETRIEVAL BASED ON FEATURE FUSION THROUGH MULTIPLE LINEAR REGRESSION ANALYSIS," ICTACT JOURNAL ON IMAGE AND VIDEO PROCESSING, AUGUST, pp. 1066-1071, 2015.

[8] A. Saini and S. Singh, "Evaluation of CBIR System by Using Low Level Features of an Image," IJSETR , pp. 1951 1954, 2016.

[9] A. Khokher and. R. Talwar , "Content-based Image Retrieval: Feature Extraction Techniques and Applications," Proceedings published in International Journal of Computer Applications ${ }^{\circledR}$ (IJCA), pp. 9-14, 2012.

[10] S. Saurav, P. Belsare and S. Sarkar, "Holistic Correlation of Color Models, Color Features and Distance Metrics on Content-Based Image Retrieval," nternational Research Journal of Engineering and Technology (IRJET) , pp. 39 -43, 2015.

[11] M. Ramana, S. Radha, E. Reddy and E.Sreenivasa, "CONTENT BASED IMAGE RETRIEVAL (CBIR) USING ADAPTIVE GROUND TRUTH COMPOSITION," International Research Journal of Engineering and Technology (IRJET) , pp. 979-983, 2015.

[12] S. Dinkar and K. Lahre, "Image Retrieval Based On Color and Texture Features Modification in Watermarking Technique," International Research Journal of Engineering and Technology (IRJET), pp. 1345-1351, 2015.

[13] P. Malode and S. V. Gumaste, "A Review Paper on Content Based Image Retrieval," International Research Journal of Engineering and Technology (IRJET), pp. 883-885, 2015.

[14] S. Yadav, S. Varne, N. Jadhav, . S. Powar and P. Patil, "Improved Accuracy of Image Retrieval by Using K-CBIR," International Research Journal of Engineering and Technology (IRJET), pp. 2343-2345, 2016.

[15] S. Barkund and S. Sonkamble, "Search-Based Face Annotation with CBIR and Clustering-based Algorithm," International Research Journal of Engineering and Technology (IRJET) , pp. 2204-2208, 2016.

[16] K. D. Prasad, K. Manjunathachari and M. N. Giriprasad, "FOURIER TRANSFORM BASED SALIENCY DETECTION FOR SKETCH BASED MAGE RETRIEVAL SYSTEMS," IJESR, pp. 608-614, 2015.

[17] S. G. Jagbir , R. Singh, P. Palta, T. Sharma and G. Goel, "CBIR of Trademark Images in different color spaces using XYZ and HSI," Journal of Network Communications and Emerging Technologies (JNCET) , pp. 84-91, 2016. 
[18] D. Giveki, A. Soltanshahi, F. Shiri and H. Tarrah, "A New Content Based Image Retrieval Model Based on Wavelet Transform," Journal of Computer and Communications, 2015,, pp. 66-73, 2015.

[19] Prachi A. Gaidhani and Bagal S. B, "Survey paper on Sketch Based and Content Based Image Retrieval," International Journal of Science and Research (IJSR), pp. 2201-2206, 2015.

[20] Syed Hamad Shirazi , Noor ul Amin Khan , Arif lqbal Umar, Muhammad Imran Razzak, Saeeda Naz and Bandar AlHaqbani , "Content-Based Image Retrieval Using Texture Color Shape and Region," International Journal of Advanced Computer Science and Applications,, pp. 418-426, 2016.

[21] Aboli W. Hole and Prabhakar L. Ramteke, "Design and Implementation of Content Based Image Retrieval Using Data Mining and Image Processing Techniques," International Journal of Advance Research in Computer Science and Management Studies , pp. 219-224, 2015.

[22] Priyanka B. Kamdi and P. Kulurkar, "Data Mining Approach for Image Retrieval in Multimodal Fusion Using Frequent Pattern Tree," IJARCSMS , pp. 95-105, 2015.

[23] Rinku Avinash Saoji and M. K. Kodmelwar, "Survey on CBIR using Halftoning BTC," International Journal of Advance Research in Computer Science and Management Studies , pp. 42-45, 2016.

[24] A. S. GOMASHE and R. R. KEOLE, "A Novel Approach of Color Histogram Based Image Search/Retrieval," International Journal of Computer Science and Mobile Computing , pp. 57-65, 2015.

[25] A. Basu, "Shape Based Image Representation and Retrieval," International Journal of Emerging Research in Management \&Technology, pp. 81-86, 2015.

[26] M. Barasia and . S. Lade, "Privacy Based Image Retrieval Using Visual and Textual Features," IJERMT, pp. 26-31, 2015 . 